\title{
An Analysis of Saxophone Training Methods in Western Universities
}

\author{
Wei Han \\ School of Music, Jimei University, Fujian, China, 361000 \\ Keywords: Saxophone music; correct procedures; basic training \\ Abstract: Saxophone music is beautiful and beautiful, and its friends are more and more in \\ China. Learning from saxophones must begin with rigorous basic training. It must be \\ trained and trained according to scientific methods and correct procedures. I hope this \\ article can help Sakes enthusiasts.
}

\section{Introduction}

In saxophone performance training, whether the training skills are appropriate or not depends largely on the correctness of the Saxophone's pronunciation, and it also directly affects the pitch and timbre. Therefore, learning how to perform saxophone must be performed with persistence.

\section{Mouth training}

Since physiological differences are objective, there are differences in how many heads and how many vibration points each saxophone learner has, and it is necessary to repeatedly search for the best vibration points. The more scientific and convenient approach is: The practitioner first starts playing the saxophone with a small number of mouthpieces, and then uses the mouthpiece to perform the blowing practice a little at a time. When he is unable to control the noise, he will explore the direction again. After repeated searches such as these, the practitioner can finally find the point that can produce the best sound, which is its best vibration point. Then, the practitioner must explore again and again to find the most suitable elastic point for his mouth - first play the saxophone with a relatively loose mouth, and then gradually play with the tighter and tighter mouth shape until no sound can be played. Look in the opposite direction again to find out the most relaxing point of your voice. Practice has proved that if you can grasp the best point of vibration, grasping the most ideal elastic point of the mouth, will help the practitioner to accurately master the saxophone performance skills, especially to facilitate their learning and master the super-treble performance skills[1].The formula of mouth training skill is as follows.

$$
G(m)=\sum_{i=1}^{m} \lambda_{i} / \sum_{k=1}^{p} \lambda_{k}
$$




\section{Breathing training}

In the whole performance of Saxophony, breathing is the most basic and most important skill, which directly determines the performance level and the success or failure of the performance. The so-called breathing, in fact, is the support of the breath, is the driving force of the saxophone. In saxophone performance, the control of breathing techniques is very difficult, and it is more difficult than playing bows in string performances.

This is related to the saxophone's pronunciation, pitch, and bass. Saxophone's breathing techniques include a wide range of training. Breathing techniques that learners must master include the control of timbre, intensity, training, and training of mid-tempo accent and weak sounds. The division of the length of the phrase, as well as the training of vibrato control, emotional expression of phrases, etc. If the performer lacks the correct breathing method, not only does the performance fail, but it also affects the health of his respiratory organs. In summary, the main advantages of the advantages of chest and abdomen combined breathing method are: First, the exhaled air flow is stronger. Using the chest and abdomen joint breathing method, the airflow can be exhaled through the strength of the abdominal muscles, so the airflow intensity is large enough to satisfy the needs of saxophone playing. The general breathing method only passes through the muscles of the chest and is not strong enough to provide enough air flow support for performance. Followed by more stable air, high control. To accurately and elegantly play saxophone, it is necessary to effectively control the strength of the air flow and ensure the stability of the air flow. The chest and abdomen combined breathing method can use the abdominal muscles to strengthen the control, and the air flow is more stable and easier to control. When the phrase needs strong or weak changes, the player can deal with it easily and easily. The third is to effectively protect the performer's respiratory organs[2].

If you want to be familiar with chest and abdominal joint breathing, you need to master certain skills and you need to practice it with perseverance. The specific training method is as follows: The practitioner uses the upper teeth to bite the mouthpiece and the lips to wrap the mouthpiece. When inhaling, use the mouth and nose to breathe into the lungs. At the same time, the chest, both sides, around the waist, the abdomen must naturally expand. Studies have confirmed that when the diaphragm is lowered by $1 \mathrm{~cm}$, the volume of the gas can be expanded by $250-300 \mathrm{~cm} 3$. If a strong inhalation is performed, the diaphragm can generally be lowered by $3-4 \mathrm{~cm}$. Because the chest and abdomen joint breathing method is based on chest muscles, abdominal muscles at the same time coordinated movements, you can maximize the amount of inspiration. Moreover, during the performance, the guest controls the inspiratory volume according to the needs of the performance of the work, and it is easy to interpret the music easily, elegantly and freely, and the player is not likely to feel tired. Saxophone learners want to practice the role of the abdomen at the same time as the thoracic cavity does not change. The chest and abdomen joint breathing method has been widely used in saxophone performances. It is the basis for playing saxophone, and it is also a ladder for enhancing saxophone performance techniques and timbres. A person who wants to change his breathing habits needs a long process and usually needs patience and perseverance. During the initial learning phase, breathing should be taken out of the instrument until the breathing is well controlled.

\section{Long tone training}

Saxophone learners are gradually mastering the chest and abdomen joint breathing method. After they can breathe and play more freely, they should practice the application of long-sound reinforcement chest-abdomen combined breathing. The long tone is a good way to practice the breath - it is the most basic practice of Saxophone, or the highest level of practice. Training long 
strings, scales, and chromatic scales is of great value in the performance of saxophones. The most frequently encountered problems in performance can be solved, and the timbre will gradually become beautiful and melodious[3].The effect of long tone training changes as shown below.
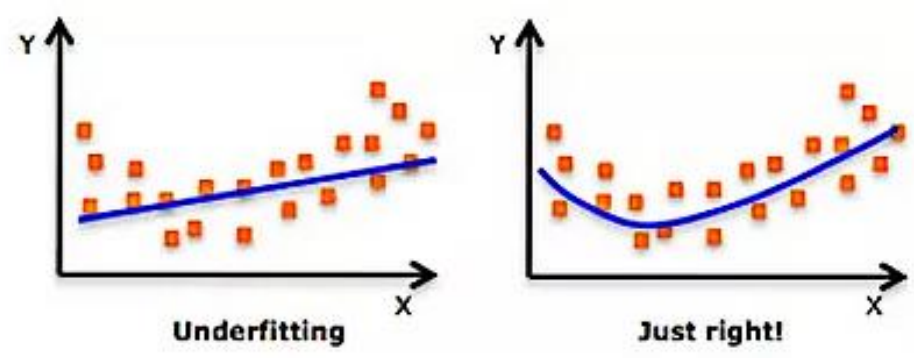

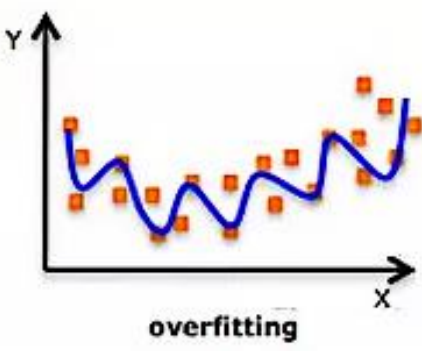

Fig.1 Effect of long tone training changes

The correct breathing and good breath for playing the hyperbaric sounds need to be realized by practicing long sounds. Because it is for playing super high notes, the practice of long-distance sounds has changed from the normal long-distance sounds. The specific method is: Take a deep breath (note that it must be sucked into the abdomen). Exhausted gas. During the performance process, attention should be paid to strong and weak changes, fluctuations in the high and low sounds. Every sound that the practitioner blew out must strive to stabilize at the correct pitch while listening to his own ear. During this process, attention should be paid to the constant adjustment of the muscles, mouth, throat and breath of the lips. For beginners to perform long-range exercises, you can start with the $\mathrm{C}$ sound, take a deep breath and then slowly exhale, with a steady flow of air to keep the sound continuous, making an octave back and forth (up and down). The speed can be slightly faster at the beginning, ensuring that one octave can be completed in one breath. Blowing tones is also a long-sounding practice with good results: When practicing, it does not perform octave, but only for single tones. Beginners can practice timing, that is, the duration of each recording of a single tone, the tone continues to reach one minute. In the above, the practice of long-range practice basically met the requirements of the general track, and there was no problem with the breath.

\section{Fuming training}

In saxophone performance, muzzle is often used as a demonstration of dazzling, and therefore plays a very important role in the performance. Vomiting is divided into tongue vomiting and air vomiting, and beginners should first master tongue vomiting. To perform a voicing exercise, you can start with the $\mathrm{C}$ tone first, blow a tone, and use your tongue to touch the mouthpiece in the mouth to complete the murmur. The exercises here can be divided into two types. One is the simplest and most basic one-tone tune. Taking $2 / 4$ beat as an example, it is practiced at a speed of 120 beats per minute. The first shot is the middle one, that is, one beat. Tones of eight-note notes, two beats followed by a long beat of two beats, then one beat of four sounds, that is, sixteenth-note sounds, the same two rows later with a two-tempo long sound, And so on with thirty-two notes. The second is to add pitch during the above exercises, and then make an octave back and forth. This kind of exercise can not only improve the speed of the tune, but also can coordinate the coordination of hands and mouth. What needs special emphasis is that we must pay attention to the accuracy and clarity of each sound, and then proceed to the new practice[4]. Tune training beat diagram is as follows. Red, green and blue represent the distribution of three beats. 


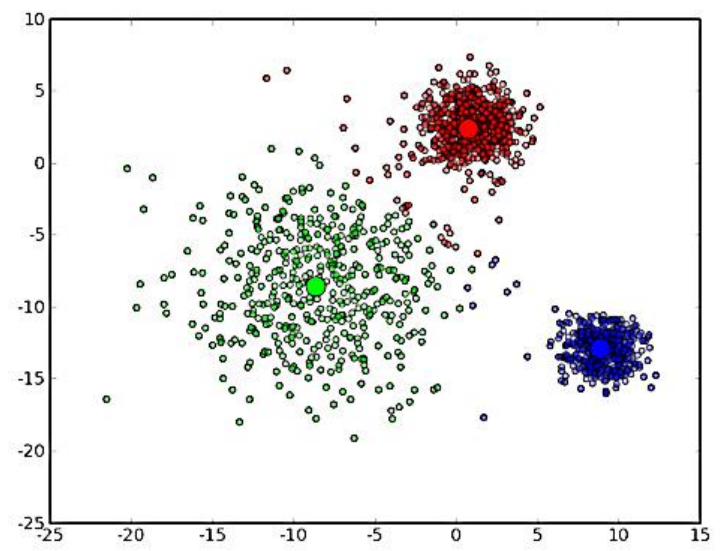

Fig.2 Tune training beat diagram

\section{Harmonic Training}

Different instruments, different vibration modes will produce different sounds. Saxophone's pronunciation is caused by the airflow exhaled by the player forming an air column in the tube, which is generated by the vibration of the impact. Sax and other brass instruments do not change the fundamental sound of the same finger in the same tube, and then rely on certain changes to blow out sounds that are octaves high, 12 degrees high, and 2 degrees high. This is the reason that the air column in the tube is under some kind of pressure. The result of segmented vibration is its overtone.

Overtones play a greater role in the performance of saxophone music, so we must strengthen its training and mastery. When performing harmonic exercises, you must try repeatedly to determine the correct match of factors such as mouth pressure, breath support, and posture of the larynx. The increased tension of the mouth should not be confused with the bite and pressure, because the latter can only play a minor role in the breath in addition to causing lower lip pain. The increased support must come from the muscles surrounding the lips, especially the muscles at the corners of the mouth. The increased tension slightly closes the tip, which requires a higher airflow rate to vibrate the tip at the desired speed.

Before the beginning of the harmonic exercises, learners must first understand the features of the harmonic series and follow a gradual, slow-to-fast process. Don't use your tongue to speak at this time, but use a breath to hit the tip.

\section{Summary}

In addition to perseverance and diligence, the key to practicing saxophone skills is to use the brain. The learners must think regularly while practicing. They should always contact the masters' classic performances. The sweat and wisdom are the ladder to success.

\section{References}

[1] Ho-Don Yan,Yu-Chen Kuo,Shan-Yu Chen. Entrepreneurship and an Apprentice-based Cluster: The Evolution of Houli's Saxophone Cluster in Taiwan[J]. Global Economic Review,2011,40(4).

[2] Pablo E. Riera, Martin Proscia,Manuel C. Eguia. A Comparative Study of Saxophone Multiphonics: Musical, Psychophysical and Spectral Analysis[J]. Journal of New Music Research,2014,43(2).

[3] Cho, Chin,Suh, Kim,Kim,Ye,Lee,Kim,Seo,Na. Postmorbid learning of saxophone playing in a patient with frontotemporal dementia[J]. Neurocase,2015,21(6).

[4] Chun-Yan Li, Zheng Guo,Zhaohui Wang. TGF $\beta$ receptor saxophone non-autonomously regulates germline proliferation in a Smox/dSmad2-dependent manner in Drosophila testis[J]. Developmental Biology,2007,309(1). 